\title{
SAR DISTRIBUTION IN HUMAN BEINGS WHEN USING BODY-WORN RF TRANSMITTERS
}

\author{
Andreas Christ ${ }^{1, *}$, Theodoros Samaras ${ }^{2}$, Esra Neufeld ${ }^{1,3}$, Anja Klingenböck ${ }^{1}$ and Niels Kuster ${ }^{1,3}$ \\ ${ }^{1}$ IT'IS Foundation, Zeughausstr. 43, CH-8004 Zürich, Switzerland \\ ${ }^{2}$ Radio Communications Lab, Aristotle University, GR-54124 Thessaloniki, Greece \\ ${ }^{3}$ Swiss Federal Institute of Technology Zürich (ETHZ), Rämistr. 101, CH-8092 Zürich, Switzerland
}

\begin{abstract}
This study analyzes the exposure of the human torso to electromagnetic fields caused by wireless body-mounted or handheld devices. Because of the frequency and distance ranges from $30-5800 \mathrm{MHz}$ and 10 to $200 \mathrm{~mm}$, respectively, both near-field and far-field effects are considered. A generic body model and simulations of anatomical models are used to evaluate the worst case tissue composition with respect to the absorption of electromagnetic energy. Both standing wave effects and enhanced coupling of reactive near-field components can lead to a specific absorption rate (SAR) increase in comparison to homogeneous tissue. In addition, the exposure and temperature increase of different inner organs is assessed. With respect to compliance testing, the observed SAR enhancement may require the introduction of a multiplication factor for the spatial peak SAR measured in the liquid-filled phantom in order to obtain a conservative exposure assessment. The observed tissue heating at the body surface under adiabatic conditions can be significant, whereas the temperature increase in the inner organs turned out to be negligible for the cases investigated.
\end{abstract}

\section{INTRODUCTION}

Owing to the novel wireless technologies, which have been introduced in recent years, the exposure of the human body to radio frequency (RF) electromagnetic fields has been constantly increasing. The basic absorption mechanism of RF energy in the human body is described in Kuster and Balzano ${ }^{(1)}$, and substantial research work has been performed to characterize the exposure of the human head due to cell phone radiation ${ }^{(2,3)}$. These studies consider tissue distributions as they typically occur in the region of the head and the temporal bone or use homogeneous tissue parameters, which have been chosen such that a conservative exposure estimate can be achieved for the exposed body region. Many handheld devices, however, expose other regions of the body than the head, or they may directly be attached to the body or the limbs. These devices can use frequencies ranging up to $6 \mathrm{GHz}$, such that both near- and far-field effects must be considered for the assessment of the specific absorption rate (SAR). Therefore, the analysis of the tissue composition and distribution must consider the whole human body. The particular impact of the presence of body fat on the absorption of electromagnetic energy has repeatedly been reported in the past ${ }^{(4,5)}$. This study therefore evaluates realistic worst case exposure situations considering generic and anatomical models of the body with respect to absorption and induced tissue heating and discusses consequences on the compliance testing of body-mounted and portable wireless devices.

\footnotetext{
*Corresponding author: christ@itis.ethz.ch
}

\section{OBJECTIVES}

The main goal of this study was the characterization of the absorption of electromagnetic fields in the trunk of the human body from wireless devices operating in the immediate environment. In detail, this encompasses:

- theoretical analysis of the near- and far-field coupling effects of electromagnetic fields into body tissue considering anatomically correct tissue distribution, distances from 5 to $200 \mathrm{~mm}$ and a frequency range up to $6 \mathrm{GHz}$;

- evaluation of the worst case tissue distribution considering the characteristics of the torso;

- analysis of the SAR distribution in anatomical models of the human body in order to validate the worst case coupling effects and to assess the exposure of inner organs;

- analysis of the induced heating effects considering worst case situations and the temperature of inner organs;

- evaluation of the results with respect to existing standards for the compliance testing of wireless devices and their applicability for different organs.

\section{ABSORPTION OF ELECTROMAGNETIC FIELDS IN LAYERED BIOLOGICAL TISSUE}

\section{Generic body model}

In order to characterize the absorption in layered body tissue, a generic body model was defined consisting of planar layers of skin, fat and muscle tissue (Figure 1). Fat tissue has a very low-water content 


\section{SAR DISTRIBUTION IN HUMAN BEINGS}

and therefore significantly lower permittivity and conductivity (e.g., $\varepsilon_{r}=5.5, \quad \sigma=0.05 \mathrm{~S} \mathrm{~m}^{-1}$ at $900 \mathrm{MHz})^{(6)}$, whereas muscle tissue has a permittivity of $\varepsilon_{r}=55$ and a conductivity of $\sigma=0.94 \mathrm{~S} \mathrm{~m}^{-1}$ at the same frequency. The dielectric properties of most other body tissues with high-water content are of the same order of magnitude.

In order to determine the tissue compositions with the highest absorption, the impact of the thicknesses of the skin and fat layers on the peak spatial average SAR has been evaluated. The thickness of the skin ranged from 0.4 to $2.6 \mathrm{~mm}$ including the epidermis and the dermis. This covers an age range from newborn to $60 \mathrm{y}^{(7)}$. Fat layer thicknesses between 0 and $\lambda / 2$ were used in order to take into account all possible effects due to impedance matching in the layers. At lower frequencies, the maximum fat thickness was limited to anatomically realistic cases. A layer of muscle tissue was used to terminate the body model.



Figure 1. Generic body model as layered tissue structure with plane wave incident to the skin.

\section{SAR calculation in layered tissue}

The absorption of electromagnetic energy in the layered body tissue has been analyzed for (1) farfield conditions assuming a perpendicularly incident plane wave in a frequency range from 236 to $5800 \mathrm{MHz}$ and (2) for the near field of a $\lambda / 16-$ and a $\lambda$ /2-dipole antenna at distances between 10 and $200 \mathrm{~mm}$. The worst case tissue compositions were evaluated with respect to the $1 \mathrm{~g}$ peak spatial average SAR in the layered structure in comparison to a homogeneous lossy half-space with the dielectric properties of head tissue simulating liquid (HTSL) as defined for the compliance testing of handheld and body-mounted devices by IEC 62209 Part $2^{(8)}$

Under far-field conditions, a layered transmission line model was used to calculate the electric field in the tissues. For the near-field evaluation, the fields of the two-dipole antennas were simulated with the finite-difference time-domain (FDTD) method (Semcad X, Schmid \& Partner Engineering, Zürich, Switzerland) and the Method of Moments with layered planar Green's functions. The peak spatial average SAR was calculated over a $1 \mathrm{~g}$ cube according to the procedure described in IEEE Std C95.3 ${ }^{(9)}$. Figure 2 shows the 1 and $10 \mathrm{~g}$ peak spatial average SARs in the layered tissue as a function of the skin and the fat thicknesses normalized to the value in HTSL at $1800 \mathrm{MHz}$. An increase of 2-3 dB in comparison to HTSL has been observed for the worst case tissue distributions. Similar results have been found for the whole frequency range of interest for the tissue thicknesses as given in Table 1 (Figure 3).

Although the tissue distribution for realistic body modeling can be more complex, a detailed analysis of the plane wave absorption considering the tissue distributions at all different regions of the torso and
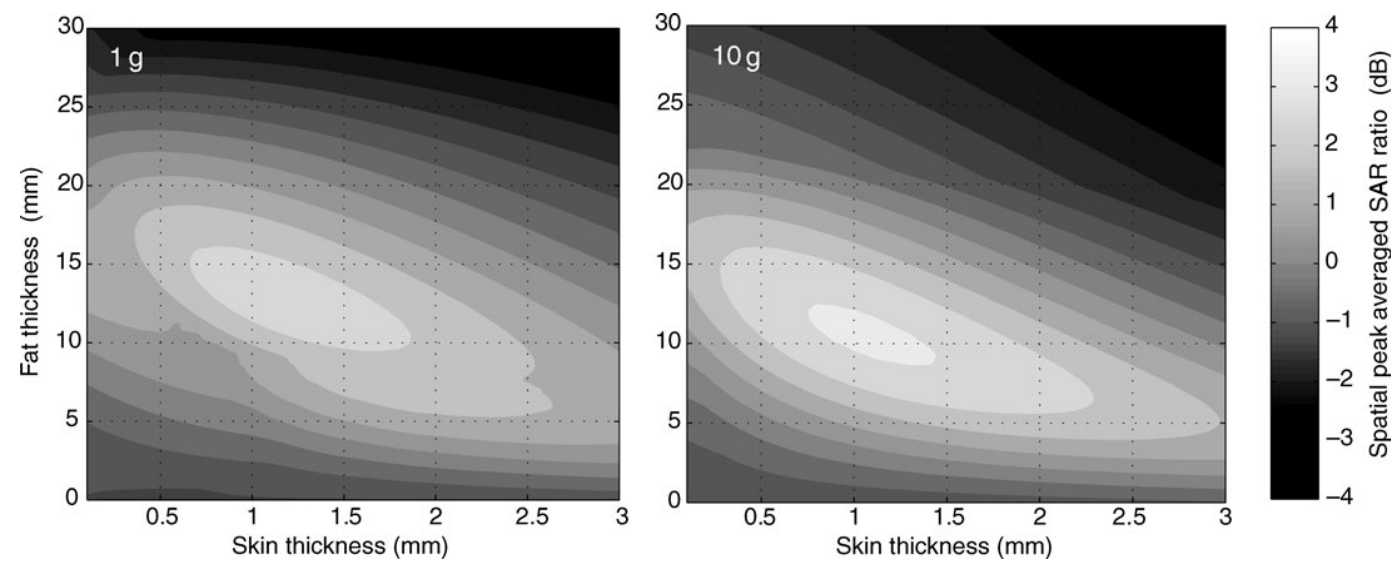

Figure 2. Ratio of the $1 \mathrm{~g}$ peak spatial average SAR in a planar structure (Figure 1) as function of the skin and fat layer thicknesses in comparison to HTSL for perpendicular incidence at $1800 \mathrm{MHz}$. 


\section{A. CHRIST ET AL}

the limbs ${ }^{(10)}$ shows results, which are in very good agreement with those obtained with the generic body model. The distribution of the spatial average SAR and the local SAR (not averaged) in anatomically correct tissue layers [skin-subcutaneous adipose tissue (SAT) - breast tissue-muscle] is shown in Figure 4. Owing to the comparatively high losses, the main contribution to the spatial average SAR occurs in the skin layer. If the sequence of tissue layers is terminated with muscle tissue, the reflections will lead to a strong increase in the skin layer ( $>4 \mathrm{~dB}$ for the case shown in Figure 4$)$. The peak spatial average SAR in a cubic volume of layered tissue is $\sim 3 \mathrm{~dB}$ higher than in HTSL. In this

Table 1. Thicknesses of the skin $\left(d_{\text {skin }}\right)$ and the fat layer $\left(d_{\text {fat }}\right)$ for maximum $1 \mathrm{~g}$ peak spatial average SAR (worst case) in the generic body model for far-field conditions.

\begin{tabular}{lcc}
\hline Frequency $(\mathrm{MHz})$ & $d_{\text {skin }}(\mathrm{mm})$ & $d_{\text {fat }}(\mathrm{mm})$ \\
\hline 236 & 2.6 & 100 \\
450 & 2.6 & 50 \\
900 & 2.6 & 24 \\
1500 & 1.5 & 15 \\
1800 & 1.3 & 13 \\
2450 & 1.2 & 7.0 \\
5800 & 0.4 & 3.8 \\
\hline
\end{tabular}

case, the high contribution of the skin layer is partially compensated by the SAT and the breast tissue. If the SAR is averaged over contiguous skin tissue instead of the cubical volume and if a sufficiently large surface of the skin is exposed, then the peak spatial average SAR in the skin layer exceeds the SAR in HTSL by $8-9 \mathrm{~dB}$.

Under near-field conditions, enhanced E-field coupling into layered tissue has been observed for low frequencies $(<450 \mathrm{MHz})$ and electrically small antennas. If the reactive energy contains significant levels of H-polarized fields ${ }^{(11)}$, the perpendicular E-field components cause highly localized absorption in the skin and also penetrate the low-permittivity fat layer. Figure 5 compares the $1 \mathrm{~g}$ peak spatial average SAR in layered and homogeneous tissue for a $\lambda / 16$ - and a $\lambda / 2$-dipole antenna in a layered and a homogeneous body model at $236 \mathrm{MHz}$. At short distances $(<25 \mathrm{~mm})$, the SAR in the layered model exceeds the one in the homogeneous model by more than $2 \mathrm{~dB}$ if the phantoms are exposed to the radiation of the $\lambda / 16$-dipole. For the $\lambda / 2$-dipole, the homogeneous body model yields a conservative SAR estimation. This effect does not depend on the actual thickness of the fat layer or on the terminating tissue. At larger distances, the above-mentioned standing wave effects lead to an SAR increase independent of the antenna type ${ }^{(12)}$.

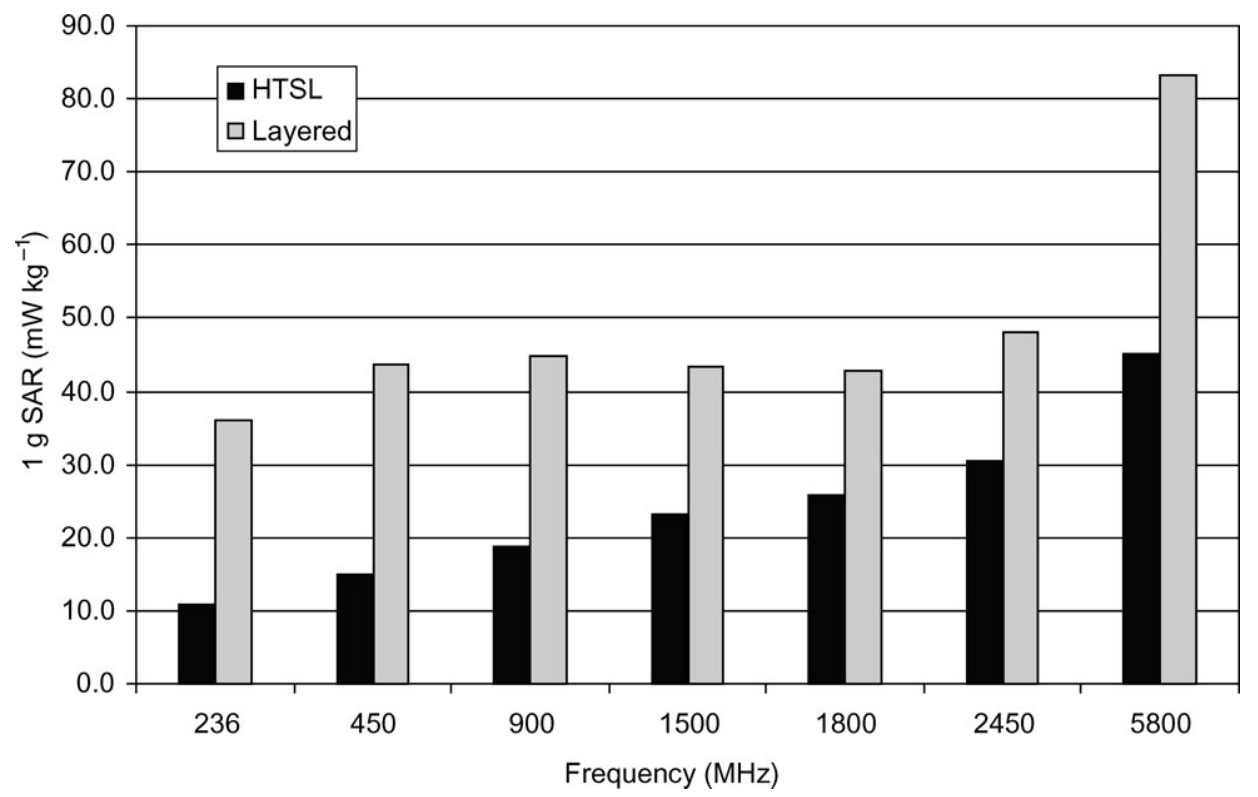

Figure 3. One-gram peak spatial average SAR in HTSL and in the generic model worst case tissue distribution (Table 1)

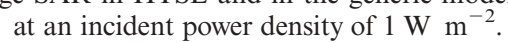




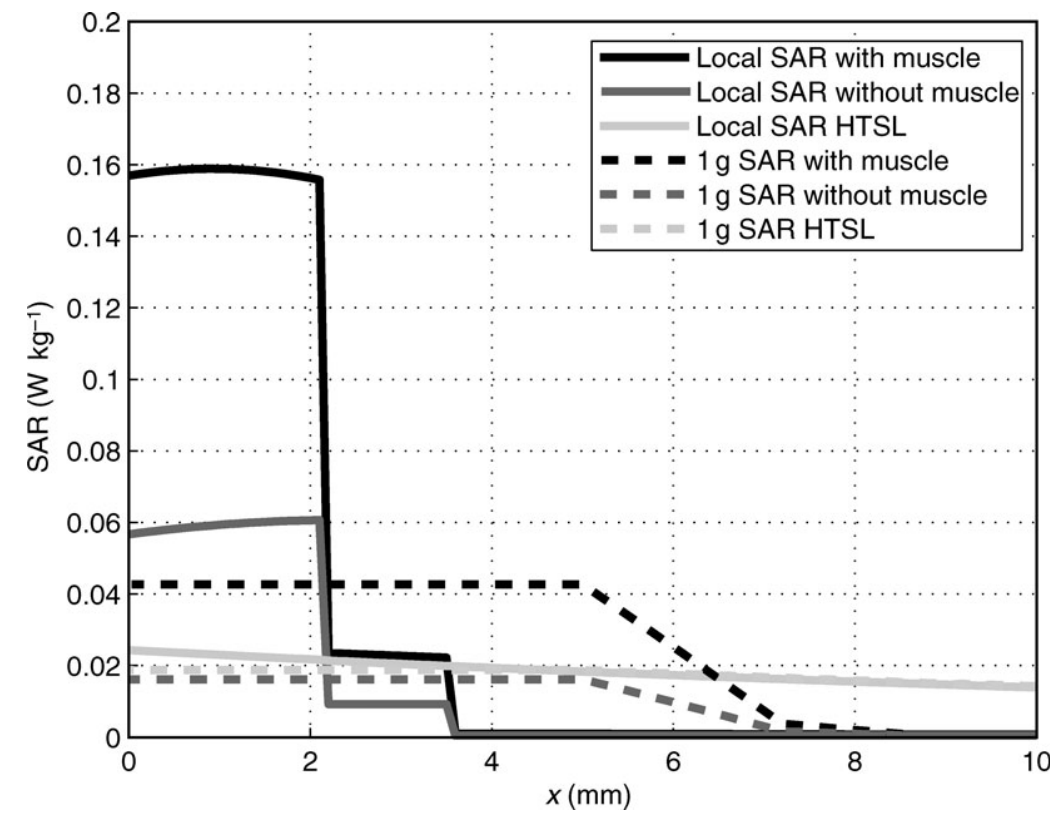

Figure 4. Local and $1 \mathrm{~g}$ peak spatial average SAR distribution in layered biological tissue under far-field conditions at $900 \mathrm{MHz}$ (2.2 mm skin, $1.4 \mathrm{~mm}$ SAT, $22.2 \mathrm{~mm}$ breast tissue with and without a terminating muscle layer) in comparison to homogeneous HTSL at an incident power density of $1 \mathrm{~W} \mathrm{~m}^{-2}$.

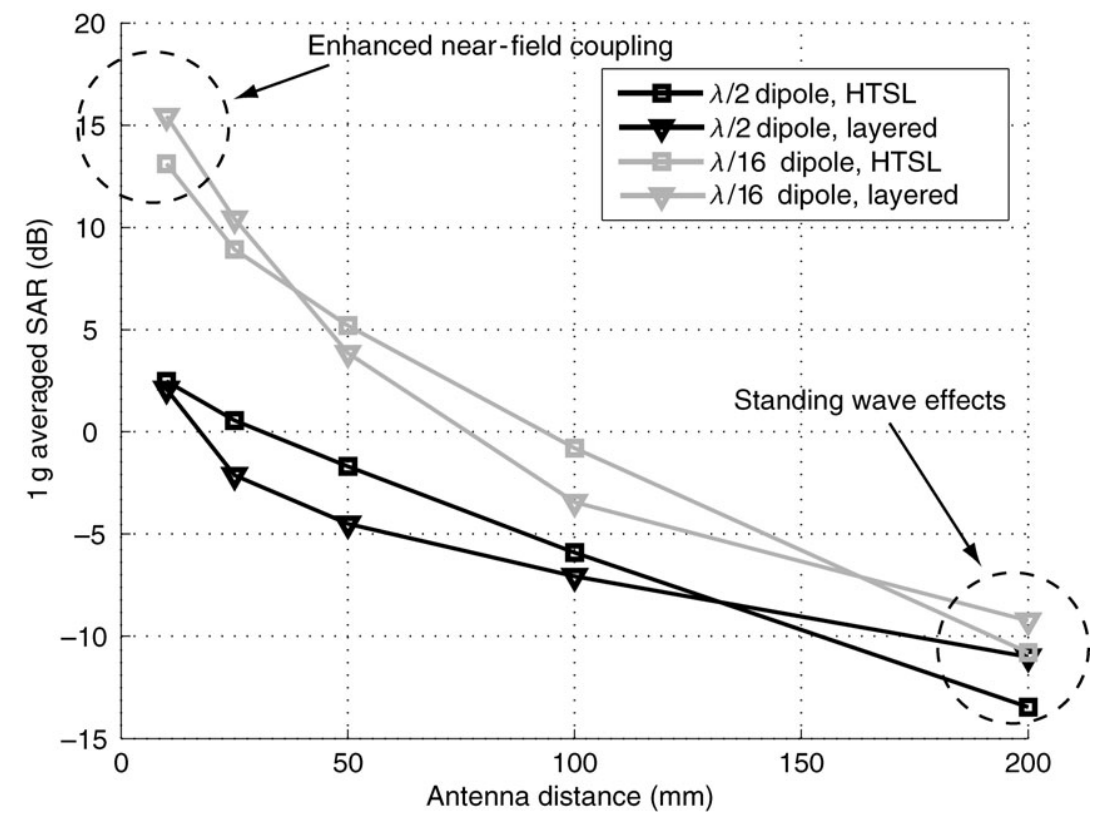

Figure 5. One-gram peak spatial average $\mathrm{SAR}$ in $\mathrm{dB}\left(0 \mathrm{~dB}=1 \mathrm{~W} \mathrm{~kg}^{-1}\right)$ at $236 \mathrm{MHz}$ for $1 \mathrm{~W}$ feeding power for a $\lambda / 16$ and a $\lambda / 2$-dipole in layered tissue (2.6 mm skin, $105 \mathrm{~mm}$ fat, muscle) and HTSL. 


\section{A. CHRIST ET AL.}

EXPOSURE OF ANATOMICAL MODELS OF THE HUMAN BODY

\section{Standing wave effects in an anatomical body model}

An anatomically correct whole-body model of an adult male of a body weight of $90 \mathrm{~kg}$ and a height of $1.80 \mathrm{~m}^{(13)}$ was used for the excitation of the standing wave effects with anatomically correct tissue distribution. A location of the abdominal wall with appropriate tissue distribution was exposed to the radiation of a $\lambda / 2$-dipole at a frequency of $1800 \mathrm{MHz}$. The distance between the antenna and the skin surface was $50 \mathrm{~mm}$ (Figure 6). Figure 7 shows the SAR distribution in the body for both anatomical and homogeneous tissue distributions when irradiated with the dipole. A significant increase in the SAR in the skin layer can be observed for the anatomically correct tissue distribution. The peak spatial average SAR in the layered model is $1.6 \mathrm{~dB}$ higher than in the homogeneous
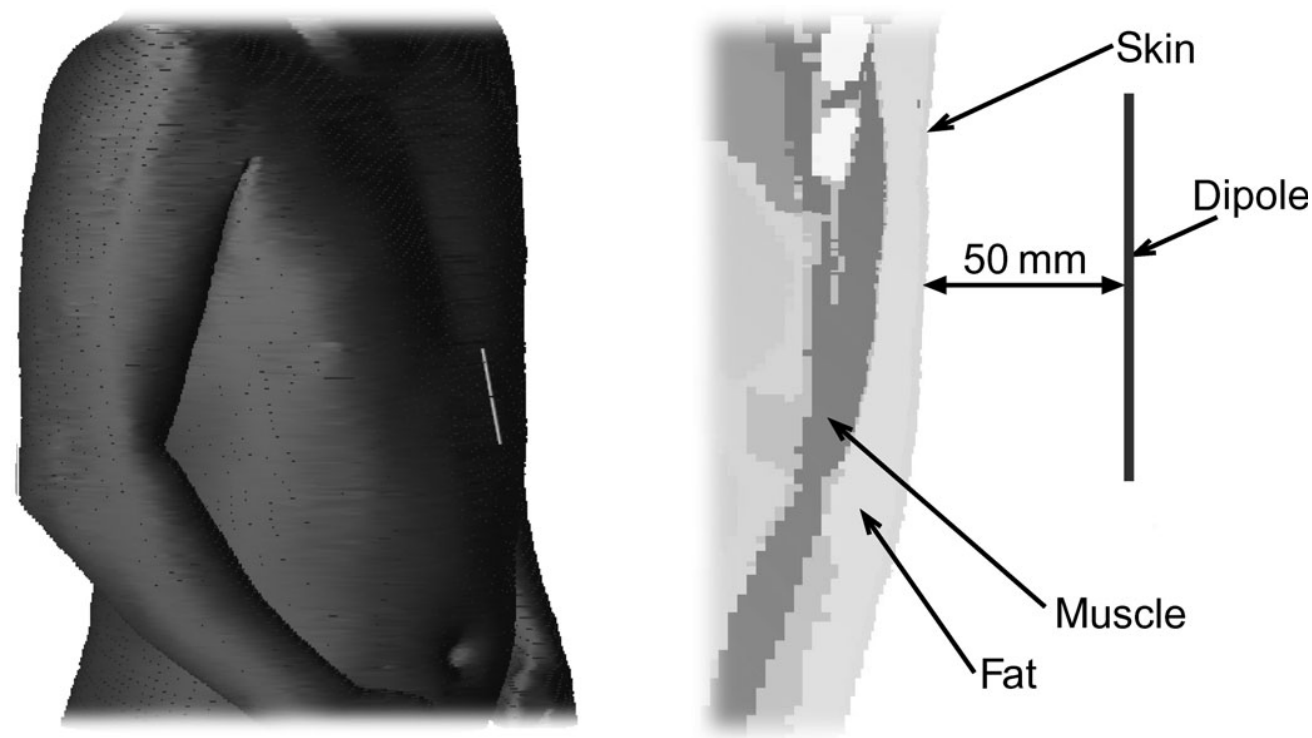

Figure 6. Positioning of the $\lambda / 2$-dipole at a distance of $50 \mathrm{~mm}$ in front of the abdominal wall. The thickness of the skin layer of the body model ranges from 2.0 to $3.5 \mathrm{~mm}$, the thickness of the fat layer from 7 to $15 \mathrm{~mm}$.
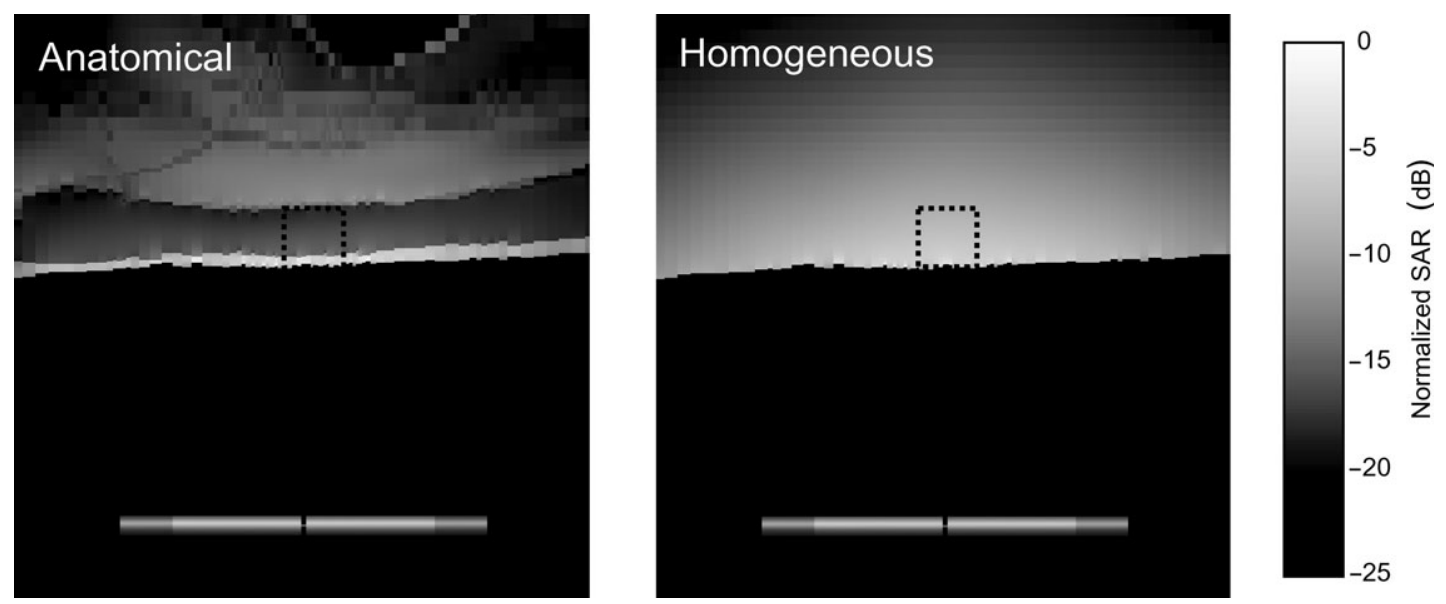

Figure 7. SAR distribution in the human body when exposed to the $\lambda / 2$-dipole for anatomically correct (right) and homogeneous (left) tissue distribution (1800 MHz, $50 \mathrm{~mm}$ distance between antenna and body); the squares mark the locations of the $1 \mathrm{~g}$ peak spatial average SAR. 


\section{SAR DISTRIBUTION IN HUMAN BEINGS}

model. Comparison with Figure 2 shows that the one-dimensional generic body model (see 'Generic body model') yields a very good estimate of the SAR increase in anatomically correct models.

\section{Exposure of the inner organs}

The exposure of inner organs was assessed using both $\lambda / 2$-dipole antennas and realistic models of generic portable wireless devices, e.g. a walkietalkie with helical antenna, a dual band mobile phone and a laptop computer with WLAN antenna. The frequency range from 450 to $5800 \mathrm{MHz}$ was considered. The positions of the devices and the orientations of the body models were chosen such that the distance to the closest point of the respective organ was minimized. A distance of $10 \mathrm{~mm}$ was kept between the transmitter and the surface of the skin. The exposure of the following organs was evaluated: heart, lung, liver, bile, kidney, intestine, stomach, pancreas and testicles. In addition to the adult male model (see 'Standing wave effects in an anatomical body model'), an anatomical model of a female $(1.60 \mathrm{~m}$, $53 \mathrm{~kg}$ ) was used ${ }^{(14)}$ in order to determine the exposure in breast tissue and the ovaries. Nonuniform FDTD-meshes were used in the simulation. A minimum mesh step size of $0.3 \mathrm{~mm}$ was used in the tissue layers at the exposure maximum in order to resolve thin structures, such as the skin surface, with sufficient numerical accuracy.

For all configurations, the 1 and $10 \mathrm{~g}$ peak spatial average SARs were evaluated both for the target organs/tissues and for all body tissues. As the exposure reference, the peak spatial average SAR was determined in an elliptically shaped flat phantom for all frequencies and transmitter types. The axis lengths of the phantom are 600 and $400 \mathrm{~mm}$, respectively. The phantom has a dielectric shell $\left(\varepsilon_{r}=\right.$ 3.7) of 2-mm thickness. It was filled with HTSL. The distance between the transmitters and the liquid of the phantom was $10 \mathrm{~mm}$. A detailed description of the phantom can be found in IEC 62209 Part $2^{(8)}$.

When all tissues were included in the averaging volume, the elliptical phantom yielded a conservative exposure estimate of the peak spatial average SAR in the anatomical body models within a range of $0-5 \mathrm{~dB}$. This mainly depended on the local tissue composition at the exposed locations, e.g. on the amount of fat tissue in the immediate environment of the antenna feedpoint. A strong correlation with the curvature of the body could not be observed. This is in agreement with the findings of 'Absorption of electromagnetic fields in layered biological tissue', since the SAR increase due to standing wave effects or enhanced E-field coupling does not occur for a distance of $10 \mathrm{~mm}$ and frequencies of $\geq 450 \mathrm{MHz}$. The main parameter, which determines the exposure of the inner organs, is their distance to the surface of the body. The highest values of the peak spatial average SAR were observed, e.g. in the stomach and in the intestine, because these are very close to the abdominal wall of the body model used in the simulations. At higher frequencies, the exposure of the inner organs drops considerably due to the reduced penetration depth. A general correlation of the exposure and the organ type, however, cannot be given, because their positions in the body depend on the individual properties of the exposed subject. An increased susceptibility of particular organs could not be observed and had not been expected.

Although the number of available anatomical models is too small for a detailed uncertainty analysis, the findings show that the maximum SAR always occurs close to the body surface and that the position of the organs in the body, i.e. their distance to the surface is the most relevant factor with respect to their exposure.

\section{THERMAL EXPOSURE OF THE TORSO}

\section{Generic layered tissue model for thermal worst case analysis}

The temperature distribution in a one-dimensional layered tissue model was evaluated assuming peak spatial average SAR values of $1.6 \mathrm{~W} \mathrm{~kg}^{-1}$ in a $1 \mathrm{~g}$ cube $^{(15)}$ and $2.0 \mathrm{~W} \mathrm{~kg}^{-1}$ in a $10 \mathrm{~g}$ cube $^{(16)}$. Planar models can be considered as sufficient approximations for cylindrical or spherical structures if their diameter is not less than $150 \mathrm{~mm}^{(17,18)}$. Sequences and thicknesses of the tissue layers were chosen according to the worst case compositions determined in Christ and co-workers ${ }^{(10,19)}$. For the temperature evaluations, an FDTD implementation of the Pennes bioheat equation ${ }^{(20)}$ was solved using a mesh step size of $0.05 \mathrm{~mm}$, which is significantly below the minimum thickness of the tissue layers. Both free convection (environmental temperature of $20^{\circ} \mathrm{C}$ ) and adiabatic boundary conditions were applied at the skin tissue. Adiabatic boundary conditions correspond to the situations of complete thermal insulation. Since the objective of the temperature evaluation was the assessment of the worst case, thermoregulatory mechanisms, such as sweating or increased blood flow, have not been considered. For the innermost tissue layer, heat advection of $70 \mathrm{~W}$ per $\left(\mathrm{K} \mathrm{m}^{2}\right)$ at a temperature of $37^{\circ} \mathrm{C}$ has been assumed. The simulations were first run without RF energy until a stable basal temperature was reached.

The temperature distributions were evaluated after 6 and 30 min of simulated exposure time. In particular for convective boundary conditions, the exposure 



Figure 8. Maximum temperature rise for free convection (left) and adiabatic boundary conditions (right) for anatomical worst case compositions ${ }^{(10)}$ for SAR limits of $1.6 \mathrm{~W} \mathrm{~kg}^{-1}\left(1 \mathrm{~g}\right.$, IEEE) and $2.0 \mathrm{~W} \mathrm{~kg}^{-1}(10 \mathrm{~g}$, ICNIRP).

interval of 6 min turned out not to be sufficient for the assessment of the maximum temperature increase. After $30 \mathrm{~min}$, all cases had reached steadystate conditions. The maximum increases in the temperature in the layered tissue structures are shown in Figure 8 for free convection and adiabatic boundary conditions. In all investigated cases, the maximum increase was observed in the skin layer or immediately behind it. This corresponds to the location of the SAR maximum, which is located in the skin layer due to the standing wave effects for the worst case tissue distributions. Although a maximum increase of $0.8^{\circ} \mathrm{C}$ could be observed for free convection, the temperature in the skin layer can rise by up to $3.5^{\circ} \mathrm{C}$ for adiabatic boundary conditions. Comparison of the temperatures for the two exposure limits of $1.6 \mathrm{~W} \mathrm{~kg}^{-1}$ in a $1 \mathrm{~g}$ cube (IEEE) and of $2.0 \mathrm{~W} \mathrm{~kg}^{-1}$ in a $10 \mathrm{~g}$ cube (ICNIRP) shows that the $1 \mathrm{~g}$ limit represents the more conservative approach. The obstruction of convection at the skin layer always leads to a significant increase in the tissue heating, if no other thermoregulatory mechanisms are taken into account.

\section{Temperature increase in anatomical body models}

The temperature increase in the inner organs of the anatomical body models was analyzed for the positions for which the SAR distributions were evaluated for exposure with the $\lambda / 2$-dipole (see 'Exposure of the inner organs'). The feedpoint power of the antenna was chosen such that the $10 \mathrm{~g}$ peak spatial average SAR of the exposed area reached the limit of $2 \mathrm{~W} \mathrm{~kg}^{-1}$. The temperature increase was evaluated for a frequency of $450 \mathrm{MHz}$, because only tissues close to the body surface contribute to the averaging volume for the exposure limit, and the induced heating of the inner organs will drop as soon as the frequency is increased. Both free convection (environmental temperature of $20^{\circ} \mathrm{C}$ ) and adiabatic boundary conditions were used. In order to reach thermal equilibrium, the anatomical models were first simulated for $40 \mathrm{~min}$ without exposure. They were then exposed for another $40 \mathrm{~min}$.

Since the tissue distribution at the exposed locations is different from the worst case structures discussed in 'Generic layered tissue model for thermal worst case analysis', the maximum heating does not occur in the skin. For the different configurations, the maximum temperature increase was generally observed at locations between 8 and $15 \mathrm{~mm}$ underneath the body surface in the muscle or SATlayer. For free convection, an increase between 0.16 and $0.23^{\circ} \mathrm{C}$ was observed. Since the maximum does not occur at the body surface, the differences to adiabatic boundary conditions were minor. The maximum temperature rise observed here was $0.31^{\circ} \mathrm{C}$. Both for convective and adiabatic boundary conditions, no values $>0.11^{\circ} \mathrm{C}$ were observed for the heating of the inner organs. Again, the induced heating strongly depends on the distance of the organ from the body surface and of the distance to the transmitter.

\section{SUMMARY AND CONCLUSIONS}

This study investigated the exposure of the torso caused by wireless transmitters operating in its immediate environment. Because of the large frequency range, which can be used by these transmitters $(30 \mathrm{MHz}-5800 \mathrm{MHz})$, both near- and far-field effects were considered. Both SAR and temperature distribution were analyzed using generic approaches and realistic models of the human body and the 


\section{SAR DISTRIBUTION IN HUMAN BEINGS}

transmitters. The results showed that the absorption of electromagnetic fields in layered body tissue may increase with respect to homogeneous tissue or tissue simulating liquids. This is due to effects whose great extent had not been anticipated or which had not yet been observed: (1) increased penetration of reactive near-field components for frequencies $<450 \mathrm{MHz}$ and electrically small antennas, and (2) standing wave effects for far-field-like exposure. The observed maximum SAR increase was $\sim 5 \mathrm{~dB}$ in comparison to the standardized tissue simulating liquids. These effects cannot be reproduced with homogeneous dosimetric phantoms. The exposure of different inner organs was assessed. Increased absorption in particular organs was not observed. The constitutive factor for the exposure of an organ is its position in the body. In all observed cases, the peak spatial average SAR at the surface of the body is larger than that of the inner organs.

The temperature increase in body tissue due to exposure to a $10 \mathrm{~g}$ peak spatial average SAR of $2 \mathrm{~W} \mathrm{~kg}^{-1}$ can reach more than $3.5^{\circ} \mathrm{C}$ under worst case conditions (one-dimensional model with adiabatic boundary conditions, maximum absorption, no thermal regulation). This significantly exceeds the order of magnitude previously assumed. For free convection, a maximum increase of $0.8^{\circ} \mathrm{C}$ was observed. The temperature increase in the anatomical phantom reaches up to $0.31^{\circ} \mathrm{C}$ for adiabatic boundary conditions. Such conditions can always occur close to the surface of the body. The temperature increase in the inner organs is negligible, if the SAR exposure limits are met.

With regard to the exposure of the inner organs, it must be considered that a detailed analysis of the uncertainty and variability of the results has not been performed because of the insufficient number of appropriate whole-body models. Nevertheless, the findings generally confirm the strong dependence of the exposure and induced tissue heating on the distance of the organ or tissue from the surface or the SAR maximum. A significant thermal load to inner body regions caused by devices, which are compliant with safety limits appears unlikely.

For the conservative compliance testing of handheld and body-mounted wireless devices with safety limits for RF-exposure, the respective standards must be adapted to take into account the effects, which were observed for the coupling of the electromagnetic fields into layered body tissue. The consortium of this study has submitted suggestions (frequency- and distance-dependent scaling factor) to the respective standardization working groups. The current standards for the compliance testing of wireless devices operated at the ear are not affected by the results of this study; the observed effects do not occur at the exposed regions of the head because of the different tissue composition.

\section{ACKNOWLEDGEMENTS}

The authors gratefully acknowledge the advice of Prof. Dr. Q. Balzano. This work was supported by the Bundesamt für Strahlenschutz, Germany, by the Swiss Commission for Technology and Innovation, by the Mobile Manufacturers Forum, Belgium, and by Schmid \& Partner Engineering AG, Switzerland.

\section{REFERENCES}

1. Kuster, N. and Balzano, Q. Energy absorption mechanism by biological bodies in the near field of dipole antennas above $300 \mathrm{MHz}$. IEEE Trans. Veh. Technol. 41, 17-23 (1992).

2. Burkhardt, M. and Kuster, N. Review of exposure assessment for handheld mobile communications devices and antenna studies for optimized performance. In Review of Radio Science 1996-1999, Stone, W.R. Ed. (Oxford: Oxford University Press) (1999).

3. Drossos, A., Santomaa, V. and Kuster, N. The dependence of electromagnetic energy absorption upon human head tissue composition in the frequency range of 300-3000 MHz. IEEE Trans. Microw. Theory Tech. 48, 1988-1995 (2000).

4. Chou, C. K. Evaluation of microwave hyperthermia applicators. Bioelctromagnetics 13, $581-597$ (1992).

5. Chuang, H.-R. Numerical computation of fat layer effects on microwave near-field radiation to the abdomen of a full-scale human body model. IEEE Trans. Microw. Theory Tech. 45, 118-125 (1997).

6. Gabriel, C., Gabriel, S. and Corthout, E. The dielectric properties of biological tissues: I. Literature survey. Phys. Med. Biol. 41, 2231-2249 (1996).

7. Snyder, W. S., Cook, M. J., Nasset, E. S., Karhausen, L. R., Howells, G. P. and Tipton, I. H. Report of the task group on reference man, first edn. (Oxford, New York, Tokyo: Elsevier Science) (1975).

8. IEC62209 Part 2. Human exposure to radio frequency fields from handheld and body-mounted wireless communication devices - human models, instrumentation and procedures, Part 2: procedure to determine the specific absorption rate $(S A R)$ for mobile wireless communication devices used in close proximity to the human body (frequency range $30 \mathrm{MHz}$ to $6 \mathrm{GHz}$ ). IEC Technical Committee 106 (Geneva, Switzerland: International Electrotechnical Commission) (2007).

9. IEEE Std C95.3. Recommended practice for measurements and computations of radio frequency electromagnetic fields with respect to human exposure to such fields, $100 \mathrm{kHz}-300 \mathrm{GHz}$ (NY: IEEE Standards Department, International Committee on Electromagnetic Safety, The Institute of Electrical and Electronics Engineers) (2002).

10. Christ, A., Klingenböck, A., Samaras, T., Goiceanu, C. and Kuster, N. The dependence of electromagnetic far-field absorption on body tissue composition in the frequency range from $300 \mathrm{MHz}$ to $6 \mathrm{GHz}$. IEEE Trans. Microw. Theory Tech. 54, 2188-2195 (2006).

11. Clemmow, P. C. The plane wave spectrum representation of electromagnetic fields (Oxford: Oxford University Press) (1996). 


\section{A. CHRIST ET AL.}

12. Christ, A., Samaras, T., Klingenböck, A. and Kuster, N. Characterization of the electromagnetic near-field absorption in layered biological tissue in the frequency range from $30 \mathrm{MHz}$ to $6000 \mathrm{MHz}$. Phys. Med. Biol. 51, 4951-4966 (2006).

13. Ackerman, M. J. The visible human project. Proc. IEEE 86, 504-511 (1998).

14. Nagaoka, T., Watanabe, S., Sakurai, K., Kunieda, E., Watanabe, S., Tak, M. and Yamanaka, Y. Development of realistic high-resolution whole-body voxel models of Japanese adult males and females of average height and weight, and application of models to radio-frequency electromagnetic-field dosimetry. Phys. Med. Biol. 49, 1-15 (2004).

15. IEEE Std C95.1. IEEE Standard for Safety Levels with Respect to Human Exposure to Radio Frequency Electromagnetic Fields, $3 \mathrm{kHz}$ to $300 \mathrm{GHz}$ (NY: IEEE Standards Department, International Committee on Electromagnetic Safety, The Institute of Electrical and Electronics Engineers) (1999).
16. International Commission on Non-Ionizing Radiation Protection. Guidelines for limiting exposure to time-varying electric, magnetic and electromagnetic fields (up to $300 \mathrm{GHz}$ ). Health Phys. 74, 494-522 (1998).

17. Cui, Z. F. and Barbenel, J. C. The influence of model parameter values on the prediction of skin surface temperature: I. Resting and surface insulation. Phys. Med. Biol. 35, 1683-1697 (1990).

18. Foster, K. R. and Erdreich, L. S. Thermal models for microwave heating and their role in standards development. Bioelectromagnetics 20, 52-63 (1999).

19. Samaras, T., Christ, A., Klingenböck, A. and Kuster, $\mathrm{N}$. Worst case temperature rise in a one-dimensional tissue model exposed to radiofrequency radiation. IEEE Trans. Biomed. Eng. 54, 492-496 (2007).

20. Pennes, H. Analysis of tissue and arterial blood temperatures in the resting human forearm. J. Appl. Physiol. 1, 93-122 (1948). 